\title{
Pandemia do novo Coronavírus (SARS-CoV-2): o protagonismo da enfermagem - uma relação do passado com o presente e perspectivas para o futuro
}

\begin{abstract}
RESUMO | Objetivos: Realizar uma reflexão sobre a pandemia global do novo coronavírus SARS-CoV-2 e o protagonismo da enfermagem neste contexto, relacionando o presente com o passado e perspectivas para o futuro. Metodologia: Estudo de reflexão teórica e descritiva em um contexto social-econômico-político. Resultados: Descreve o SARS-CoV-2 que causa a doença COVID-19; pontua também as estratégias globais para minimizar sua rápida disseminação. Relata o protagonismo da enfermagem neste contexto, por serem os profissionais que estão na linha de frente nos cuidados de controle da infecção de uma doença sem tratamento específico e por estarem do lado dos pacientes 24 horas/dia, desde os cuidados básicos aos intensivos. Acrescentem-se a estes fatores o trabalho realizado com a escassez, a ausência ou falta de qualidade e segurança dos equipamentos de proteção individual, a carência de recursos humanos, profissionais afastados do trabalho devido à COVID-19, óbitos e uma falta de visibilidade social do passado que se faz presente nesta pandemia. Conclusão: A enfermagem nesta pandemia ganhou visibilidade e protagonismo no mundo. As perspectivas para o futuro da enfermagem e a importância de se concretizarem ações políticas devem visar, portanto, ao suprimento das demandas identificadas para que se evitem os problemas do passado, abundantemente evidenciados nesta pandemia.
\end{abstract}

Palavras-chaves: Coronavírus; Enfermagem; Controle de Infecção; História da Enfermagem

\begin{abstract}
Objectives: Carry out a reflection on the global pandemic of the new coronavirus SARS-CoV-2 and the role of nursing in this context, relating the present with the past and perspectives for the future. Methodology: Study of theoretical and descriptive reflection in a social-economic-political context. Results: Describes the SARS-CoV-2 that causes COVID-19 disease; it also points out the global strategies to minimize its rapid spread. She reports the role of nursing in this context, as they are the professionals who are at the forefront of infection control of a disease without specific treatment and for being on the side of patients 24 hours a day, from basic to intensive care. Add to these factors the work done with the scarcity, the absence or lack of quality and safety of personal protective equipment, the lack of human resources, professionals away from work due to COVID-19, deaths and a lack of social visibility from the past that is present in this pandemic. Conclusion: Nursing in this pandemic has gained visibility and prominence in the world. The perspectives for the future of nursing and the importance of carrying out political actions must therefore aim at meeting the identified demands to avoid the problems of the past, abundantly evidenced in this pandemic.
\end{abstract}

Keywords: Coronavirus; Nursing; Infection Control; History of Nursing.

RESUMEN | Objetivos: Realizar una reflexión sobre la pandemia global del nuevo coronavirus SARS-CoV-2 y el papel de la enfermería en este contexto, relacionando el presente con el pasado y las perspectivas para el futuro. Metodología: Estudio de la reflexión teórica y descriptiva en un contexto socio-económico-político. Resultados: describe el SARS-CoV-2 que causa la enfermedad COVID-19; También señala las estrategias globales para minimizar su rápida propagación. Ella informa sobre el papel de la enfermería en este contexto, ya que son los profesionales que están a la vanguardia del control de infecciones de una enfermedad sin un tratamiento específico y por estar del lado de los pacientes las 24 horas del día, desde cuidados básicos hasta cuidados intensivos. Agregue a estos factores el trabajo realizado con la escasez, la ausencia de falta de calidad y seguridad del equipo de protección personal, la falta de recursos humanos, profesionales fuera del trabajo debido a COVID-19, muertes y falta de visibilidad social. del pasado presente en esta pandemia. Conclusión: La enfermería en esta pandemia ha ganado visibilidad y prominencia en el mundo. Por lo tanto, las perspectivas para el futuro de la enfermería y la importancia de llevar a cabo acciones políticas deben apuntar a satisfacer las demandas identificadas para evitar los problemas del pasado, ampliamente evidenciados en esta pandemia.

Palavras claves: Coronavirus; Enfermería; Control de Infección; Historia de la Enfermería.

\section{Patricia Cristina Cavalari de Oliveira}

Enfermeira. Mestre em Educação. Especialista em Enfermagem Pediátrica. Preceptora de Estágio do Curso de Graduação de Enfermagem da Universidade Anhembi Morumbi (UAM). SP/Brasil.

ORCID: https://orcid.org/0000-0002-9832-8615

Recebido em: 03/06/2020

Aprovado em: 03/06/2020

INTRODUÇÃO

m tempos de pandemia, o cenário
que envolve as populações e os pro-
fissionais de saúde provoca grande aflição demandando a tomada de decisões rápidas para mitigar seus efeitos. Nesta, não é diferente. Por ser causada por um novo tipo de coronavírus, denominado SARS-CoV-2, que até então não havia sido identificado em seres humanos exigiu das autoridades e dos órgãos oficiais da saúde estratégias globais para minimizar sua rápida disseminação ${ }^{(1)}$.
A enfermagem não tinha visibilidade social como a que estamos vivendo atualmente. Por este motivo, é preciso destacar que uma campanha global, iniciada em 2018, com o tema Nursing Now (Enfermagem Agora), antecipava uma ação internacional para apresentar os profissionais da categoria como os verdadeiros protagonistas da saúde no ano de 2020. Além disso, uma comemoração no dia do enfermeiro em 12 de maio de 2020 com o tema "Enfermeiros: uma voz para lide- 
rar - Enfermagem do mundo para a saúde e as comemorações do bicentenário do nascimento de Florence Nigthtingale, precursora da enfermagem moderna", contribuíram para realçar a importância da categoria ${ }^{(2,3)}$.

Cuidados de enfermagem de higiene, de conforto e de alimentação, instituídos por Florence ${ }^{(4,5)}$ no passado, base da ciência para área, evoluíram com total embasamento científico e práticas baseadas em evidências, inclusive para os cuidados intensivos, tão presentes nesta pandemia $^{(6-7)}$.

A enfermagem está na linha de frente nos cuidados de controle da infecção da doença em que inexiste possibilidade de tratamento específico. São os profissionais da enfermagem que ficam do lado do paciente 24 horas/dia, que trazem alívio dos sinais e sintomas e das alterações hemodinâmicas que são causadas no organismo pelo SARS-CoV-2 ${ }^{(6,8-9,13)}$.

No exercício de suas funções, no mundo inteiro, são marcantes os problemas que estes profissionais têm enfrentado nesta pandemia, na linha de frente no cuidado do controle da infecção causada pela doença COVID-19 devido à escassez de equipamentos de proteção individual (EPI) e à falta de recursos humanos nos serviços de saúde que aumentaram por causa dos afastamentos devido à contaminação pela doença e aos óbitos ${ }^{(8,10,13)}$.

Diante deste cenário, é relevante um estudo para realizar uma reflexão sobre a pandemia global do novo coronavírus SARS-CoV-2 e o protagonismo da enfermagem neste contexto, relacionando o presente com o passado e perspectivas para o futuro.

\section{METODOLOGIA}

Estudo de reflexão teórica e descritiva em um contexto social-econômico-políti$\mathrm{co}$, de abordagem qualitativa de análise de conteúdo de Bardin ${ }^{(11)}$, a partir de categorias pré-definidas sobre o tema, com o objetivo de realizar uma reflexão sobre a pandemia global do novo coronavírus
SARS-CoV-2 e o protagonismo da enfermagem neste contexto, relacionando o presente com o passado e perspectivas para o futuro.

O embasamento teórico foi construído em maio de 2020, considerando a circunstância inusitada de uma pandemia global por um novo agente patogênico - o novo coronavírus SARS-CoV-2 e a escassez de estudos sobre o tema, tendo significado e relevância a utilização de documentos eletrônicos de órgãos fiscalizadores do exercício profissional de enfermagem - do Conselho Federal de Enfermagem, do Conselho Regional de Enfermagem de São Paulo, e outros órgãos como o Conselho Internacional de Enfermeiras, o do Ministério da Saúde e da Organização Mundial de Saúde (OMS) e de mais três estudos/artigos selecionados em bases de dados, a PubMed Central e SciELO. Os descritores utilizados: "coronavírus", "enfermagem", "controle de infecção" e "história da enfermagem".

Seguindo as fases da análise de conteúdo de Bardin, de pré-análise, exploração do material, tratamento dos resultados obtidos, inferência e interpretação. Realizado a descrição em duas categorias temáticas: 1. A pandemia global do novo coronavírus (SARS-Cov-2) causador da doença COVID-19; 2. O protagonismo da enfermagem frente à pandemia: uma relação do presente com o passado e perspectivas para o futuro. Em seguida, é apresentada a discussão dos resultados das categorias encontradas.

\section{RESULTADOS}

Pandemia global do novo coronavírus (SARS-CoV-2) causador da doença COVID-19

Em 31 de dezembro de 2019, a Organização Mundial da Saúde (OMS) foi alertada sobre vários casos de pneumonia na cidade de Wuhan, província de Hubei, na República Popular da China. Tratava-se do mais recente tipo (cepa) descoberto de coronavírus que não havia sido identificado antes em seres humanos, um betacoronavírus que recebeu o nome de SARS-CoV-2 ${ }^{(1,9)}$.

O SARS-CoV-2 é altamente patogênico e responsável por causar síndrome respiratória e gastrointestinal, sintomas da doença denominada COVID-19. No início, muitos dos pacientes do surto na China, onde se pressupõe ter se originado o novo coronavírus, teriam tido algum tipo de vínculo com um grande mercado de frutos do mar e animais selvagens na cidade de Wuhan, sugerindo uma disseminação do vírus de animais para pessoas. Em seguida, percebeu-se que um número crescente de pacientes não havia tido qualquer exposição no referido mercado. Após inúmeras investigações, observou-se que o SARS-CoV-2 possuía uma alta e sustentada transmissibilidade de pessoa para pessoa ${ }^{(9)}$.

Os coronavírus, antes de serem considerados altamente patogênicos, circulavam em seres humanos e causavam infecções respiratórias e intestinais leves e imunocompetentes. Também são a segunda principal causa de resfriado comum (após rinovírus). Ao todo, sete coronavírus (cepas) humanos (HCoVs) já foram identificados, sendo quatro deles considerados leves: HCoV-229E, HCoV-OC43, HCoV-NL63, HCoV-HKU1 ${ }^{(12)}$.

No início do século XXI, sugiram mais dois tipos de coronavírus (betacoronavírus), que também são altamente transmissíveis e patogênicos e responsáveis por causarem síndrome respiratória e gastrointestinal nos seres humanos: o coronavírus da síndrome respiratória aguda grave (SARS-CoV) originado na China, no ano de 2002, e o coronavírus da síndrome respiratória do Oriente Médio (MERS-CoV) originado no Oriente Médio, no ano de 2012. Os dois coronavírus foram transmitidos diretamente aos seres humanos de civetas de mercado e de camelos dromedários, e acredita-se que ambos os vírus tenham se originado em morcegos ${ }^{(12)}$.

O novo coronavírus, SARS-CoV-2, o último dos sete já descobertos, apresenta um espectro clínico que varia de in- 
fecções assintomáticas a quadros mais graves. A maioria (cerca de $80 \%$ ) dos pacientes com COVID-19 pode ser assintomático ou sintomático e, aproximadamente, $20 \%$ dos casos detectados requerem atendimento hospitalar por apresentarem dificuldade respiratória, dos quais aproximadamente $5 \%$ podem necessitar de suporte ventilatório, podendo levar a óbito. Os sinais e sintomas mais comuns dessas infecções são tosse, febre, coriza, dor de garganta e dispneia. A febre pode não estar presente em alguns pacientes, como crianças, idosos, imunossuprimidos ou que tomam medicamentos para diminuir a febre ${ }^{(9)}$.

As pessoas em grupo de risco são pessoas acima de 60 anos e gestantes de alto risco, e as comorbidades mais prevalentes são também em pessoas acima de 60 anos e gestantes, pessoas com doença cardiovascular, doença renal, diabetes, doença pulmonar crônica, neoplasias, imunodeprimidos e pneumopatas. A transmissão acontece de uma pessoa doente para outra por contato próximo por meio de toque, gotículas de saliva, espirro, tosse, catarro, objetos ou superfícies contaminadas $^{(9)}$.

Acredita-se que o período de incubação do SARS-CoV-2 é de 2 até 14 dias após a exposição. Pode-se transmitir a doença durante o período sintomático e sugere-se que a transmissão também possa ocorrer mesmo sem o aparecimento de sinais e sintomas. Recomenda-se o isolamento domiciliar de todos os casos suspeitos ou confirmados de COVID-19, por 14 dias após o início dos sintomas ${ }^{(9,13)}$.

A suscetibilidade é geral, por ser um vírus novo. Sobre a imunidade não se sabe se a infecção em humanos que não evoluíram para o óbito irá gerar imunidade contra novas infecções e se essa imunidade é duradoura por toda a vida ${ }^{(9)}$.

Quanto ao tratamento da doença COVID-19, até o momento, não há medicamento específico. Há estudos científicos sobre o uso de diferentes medicamentos na esperança de se encontrar aquele que provoque a cura da doença, porém nada há de conclusivo. Em complemento ao uso desses medicamentos, medidas de suporte devem ser implementadas e um adequado manejo clínico ${ }^{(9)}$.
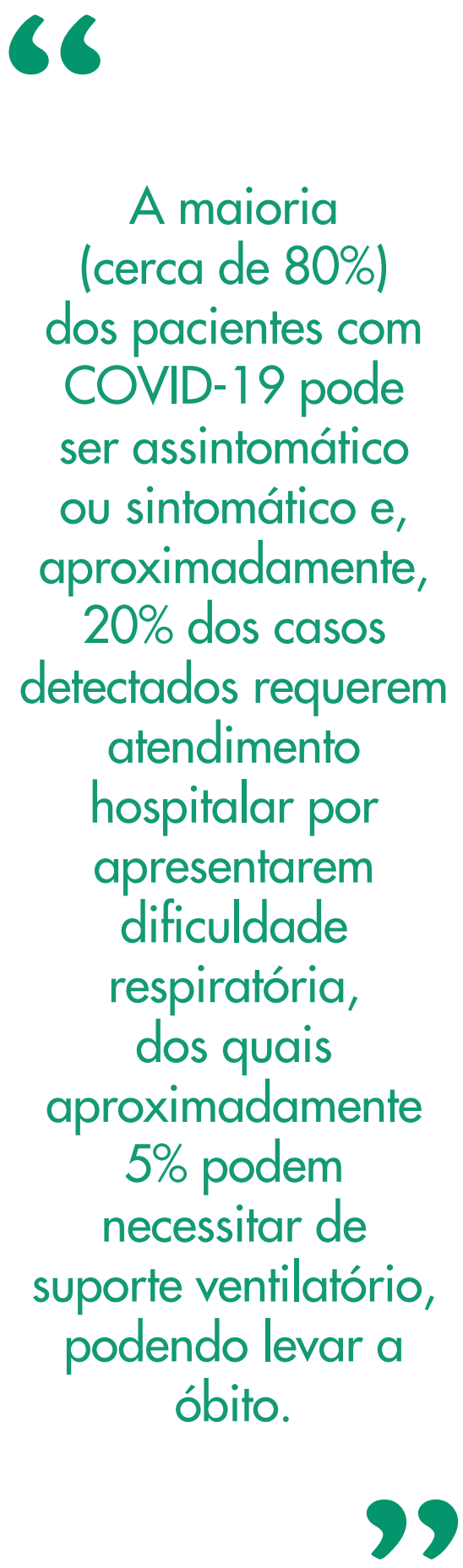

Em 30 de janeiro de 2020, a OMS declarou que o surto do SARS-CoV-2 constituía uma Emergência de Saúde Pública de Importância Internacional o mais alto nível de alerta da Organização, conforme previsto no Regulamento Sanitário Internacional. Em 11 de março de 2020, classificou a doença COVID-19 como pandemia e tem prestado apoio técnico aos países e recomendado manter o sistema de vigilância alerta, preparado para detectar, isolar e cuidar precocemente de pacientes infectados com o novo coronavírus ${ }^{(1)}$.

Em 18 de março de 2020, a OMS orientou os países no sentido de aumentarem sua capacidade de detectar casos, cuidarem de pacientes e garantirem que os hospitais tenham o espaço, suprimentos e funcionários necessários para prestar os atendimentos.

Acrescente-se a isso, a necessidade de líderes da saúde envolverem cidadãos e outros setores no apoio às ações de saúde pública para conter a situação, achatar a curva epidêmica e, assim, evitar sobrecarregar os serviços de saúde para que possam oferecer os cuidados necessários a todas as pessoas que precisam. Tudo isso para não causar o colapso dos serviços de saúde, o que indicaria uma falta de possiblidade de atender pessoas com segurança e qualidade. Sendo os profissionais de saúde a primeira linha de defesa no controle de infecção dessa pandemia, os hospitais foram incentivados a desenvolverem planos de emergência e garantirem que os esses profissionais tivessem o EPI e o treinamento necessários para prevenir a infecção ${ }^{(1)}$.

A maioria dos países adotaram essas medidas para redução da taxa de transmissão e proteção de suas populações nesta pandemia. Para além do âmbito hospitalar, observam-se medidas desde declarar estado de emergência e fechamento de fronteiras, de escolas, de comércios e de universidades, até a promoção de distanciamento social ${ }^{(1)}$.

No Brasil, em 11 de março de 2020, foi iniciado o distanciamento social com fechamento de escolas e proibição de eventos $^{(1)}$, e no dia 14 de março de 2020, 
o Ministério da Saúde atualizou e definiu medidas não farmacológicas para reduzir a possibilidade de transmissão do vírus, retardando a progressão da pandemia e reduzindo o número de casos. Essas medidas visaram atrasar o pico da pandemia e reduzir a altura do pico e diminuir a velocidade da propagação da doença permitindo uma melhor distribuição de casos ao longo do tempo e a evitar o esgotamento dos serviços de saúde e, também, para dar tempo de disponibilizar mais serviços de saúde e equipá-los com EPIs, testes laboratoriais e recursos humano. Muitos estados aderiram às medidas não farmacológicas e de distanciamento social ampliado ${ }^{(14)}$.

No Mundo, até dia 1 de junho de 2020, tivemos 6.057.853 de casos confirmados e 371.166 óbitos pelo novo coronavírus (SARS-CoV-2). No Brasil, foram 498.440 casos detectados e 28.834 óbitos $^{(15)}$.

O protagonismo da enfermagem frente à pandemia: uma relação do presente com o passado e perspectivas para o futuro

Estamos vivendo, provavelmente, um marco histórico na profissão da enfermagem, e em nível mundial. Até a chegada desta pandemia causada pelo SARS-CoV-2, a profissão da enfermagem não tinha visibilidade social como a que estamos observando atualmente, tendo em vista que a enfermagem está na linha de frente nos cuidados de controle da infecção causada pela doença COVID-19.

Mas mesmo com essa falta de visibilidade social, que se dá também pela falta de reconhecimento, autoconhecimento e valorização da própria categoria, a enfermagem evoluiu com total embasamento científico para o cuidado de prática baseada em evidências científicas ${ }^{(6-7)}$.

A enfermagem evoluiu muito desde que a precursora da profissão de enfermeira no mundo, Florence Nightingale (1820-1910), se tornou protagonista na história da enfermagem moderna. No dia 12 de maio de 2020, comemora-se seu bicentenário (200 anos). Florence marcou a história no final do século XIX atuando na Guerra da Criméia (18541856). Foi uma heroína para os soldados britânicos de quem cuidou. Desde então é exemplo para a humanidade, principalmente para a profissionalização da enfermagem, por ter modificado as condições de saúde da sociedade ${ }^{(4-5)}$.

O tempo em que Florence atuou na Guerra, no Hospital de Scutari, permitiu provar que cuidados de higiene, conforto e alimentação eram essenciais e que poderiam ajudar a resolver parte dos problemas de saúde do século XIX. Naquela época, o tratamento com medicações era raro, ocorrendo 20 anos antes de Pasteur e Koch divulgarem a teoria do germe e um século antes da criação dos primeiros antibióticos, com a exceção singular da terapia com quinino para a malária. Os médicos tinham poucos remédios para gerenciar doenças infecciosas ${ }^{(4-5)}$.

Nesta "guerra" atual, no combate ao SARS-CoV-2, os profissionais de enfermagem representam a maior categoria atuante na área de saúde, seja em instituições públicas ou nas privadas, e são os únicos profissionais presentes na assistência direta ininterruptamente junto dos pacientes. Nestas circunstâncias, estão muito mais suscetíveis à contaminação devido a sua atuação no acolhimento, detecção e avaliação das situações suspeitas de contágio do novo coronavírus quando desempenham suas funções com a capacidade técnica preconizada ${ }^{(8,10,13)}$.

No Brasil, dia 01 de junho de 2020, foi divulgado o registro de 165 óbitos e 16.904 casos de profissionais de enfermagem infectados pela doença COVID-19 ${ }^{(10)}$. O levantamento retrata o impacto das infecções causada pelo SARS-CoV-2 entre enfermeiros, técnicos e auxiliares. Esta situação se deve à escassez de EPIS, a capacitação adequada das equipes, falta de fluxos/protocolos, sobrecarga de trabalho e ao subdimensionamento das equipes ${ }^{(8)}$.

É sabido que se preconiza a utilização pelos profissionais de saúde dos EPIs, óculos de proteção ou protetor facial (face shield), máscara N95/PFF2, máscara cirúrgica, avental, luvas de procedimento e gorro (para procedimentos que geram aerossóis). Da mesma forma, os serviços de saúde devem, obrigatoriamente, disponibilizar os EPIs e fornecer capacitação para todos os profissionais de saúde ${ }^{(13)}$.

A OMS declarou em meio à pandemia que a enfermagem é sumamente importante no esforço global para o alcance dos Objetivos De Desenvolvimento Sustentável, em que se incluem a cobertura universal de saúde, saúde mental e doenças não transmissíveis, resposta a emergências, segurança do paciente e a oferta de cuidado integral e humanizado. Ao mesmo tempo, a OMS reconhece que uma agenda global para conter a epidemia deve, necessariamente, incluir esforços articulados e sustentáveis para maximizar a contribuição da força de trabalho da enfermagem e o seu papel em equipes de saúde ${ }^{(16)}$.

Diante do atual cenário caótico e incerto existente no nosso sistema de saúde, em que a enfermagem é a linha de frente na pandemia, observa-se que campanhas já vinham acontecendo para valorização dos profissionais da enfermagem como protagonistas na saúde e doença em nível mundial sem que a isso, aqui, se desse a devida importância ${ }^{(7)}$. Entre estas, o Nursing Now (Enfermagem Agora), é uma campanha global de três anos, iniciada em 2018 e que termina em 2020, e é realizada em colaboração com o Conselho Internacional de Enfermeiros (ICN) e a OMS. É uma grande ação internacional para apresentar os profissionais da categoria como os verdadeiros protagonistas da saúde no ano de $2020^{(2-3)}$.

O ICN comemora o dia internacional dos enfermeiros no dia 12 de maio, e para o ano de 2020 o tema oficial é Enfermeiros: uma voz para liderar - Enfermagem do mundo para a saúde. Nesta campanha de 2020, também se comemora o bicentenário do nascimento de Florence Nigthtingale, conforme já mencionado ${ }^{(3)}$.

A OMS, Nursing Now e ICN emitiram um importante relatório sobre a situação mundial da enfermagem em abril de 2020. Nele é identificado que 
não há enfermeiros e parteiras suficientes no mundo, e é revelado um déficit global de 5,9 milhões de enfermeiros. Mesmo deficitária, a enfermagem é o maior grupo de ocupação do setor da saúde, representando aproximadamente $59 \%$ das profissões da saúde. Neste relatório, as orientações futuras para a política da força de trabalho de enfermagem são: aumentar o financiamento para educar e empregar enfermeiros; países devem fortalecer a capacidade de coleta, análise e uso de dados da força de trabalho em saúde; verificar esses cálculos e utilizar para análises de dados do mercado de trabalho em saúde; mobilidade e a migração dos enfermeiros devem ser efetivamente monitoradas, responsáveis e geridas de forma ética, e as ações necessárias incluem o reforço da implementação do Código de Prática Global da OMS para o Recrutamento Internacional de Pessoal de Saúde, por países, recrutadores e partes interessadas internacionais; os programas de educação e treinamento de enfermeiros devem formar enfermeiros, que impulsionem o progresso, principalmente, na cobertura universal na atenção primária à saúde; as ações incluem também investimento no corpo docente de enfermagem, disponibilidade de locais de avaliação clínica e acessibilidade dos programas oferecidos para atrair um corpo discente diversificado de enfermagem; as ações incluem estabelecer e apoiar o papel de uma enfermeira sênior no governo responsável por fortalecer a força de trabalho nacional de enfermagem e contribuir para as decisões de políticas de saúde; modelos eficazes de assistência liderados por enfermeiros devem ser expandidos, para atender às necessidades de saúde da população e melhorar o acesso aos serviços de atenção primária; os formuladores de políticas, empregadores e reguladores devem coordenar ações em apoio ao trabalho digno; os países devem fornecer um ambiente propício para a enfermagem melhorando a atra- ção, implantação, retenção e motivação da equipe de enfermagem; devem propiciar níveis adequados de pessoal de segurança e saúde no trabalho; os países devem ainda planejar deliberadamente políticas e força de trabalho de enfermagem sensíveis a categoria; ações devem incluir a implementação de um sistema equitativo e neutro da categoria, remuneração entre os trabaIhadores da saúde e a garantia de que as políticas e leis que tratam da disparidade salarial entre as categorias também se apliquem ao setor privado ${ }^{(16)}$.

No Brasil, em 24 de abril de 2019, foi lançada a Campanha Nursing Now. Algumas metas estabelecidas são: investimento e fortalecimento em educação e desenvolvimento dos profissionais de enfermagem com foco na liderança; a busca pela melhoria das condições de trabalho dos profissionais de enfermagem; a disseminação de práticas de enfermagem efetivas e inovadoras com base em evidências científicas em âmbito nacional e regional ${ }^{(7)}$.

\section{DISCUSSÃO}

No mundo, a doença COVID-19 causada pelo vírus SARS-CoV-2, altamente patogênico pelo potencial índice de disseminação e sem possiblidade de tratamento especifico, tem afligido a população e estratégias globais foram necessárias para conter a disseminação da doença ${ }^{(1,9,14)}$.

Estratégias como distanciamento social ampliado, aumento de número de leitos com respiradores em hospitais, construção de hospitais de campanhas e contratação de profissionais de saúde, principalmente os de enfermagem, foram necessários para minimizar os números de óbitos causados pela doença.

Neste contexto, os profissionais de enfermagem estão na linha de frente nos cuidados de controle da infecção pela doença COVID-19. Representam a maior categoria atuante na área de saúde, seja em instituições públicas ou seja nas privadas, e têm enfrentado grandes desafios pela escassez de EPIs, falta de recursos humanos, afastamentos por alto índice de contaminação e óbitos ${ }^{(8,10,13)}$.

Constata-se que a enfermagem tem vivenciado muitos problemas nesta pandemia, a despeito das várias estratégias instituídas, pela sua atuação na atenção primária, secundária e terciária no processo de saúde e doença.

A profissão da enfermagem não tinha visibilidade social como a que estamos reconhecendo atualmente, desde a época em que a precursora da profissão da enfermagem, Florence Nightingale, na Guerra da Criméia no Hospital de Scutari, se tornou protagonista na história da enfermagem moderna, no mundo, provando que cuidados de higiene, conforto e alimentação foram essenciais para resolver parte dos problemas de saúde e doença do século XIX, em um tempo em que tratamentos com medicações eram raros para combater doenças infecciosas ${ }^{(4-5)}$.

Relacionando-se este passado descrito ao presente, os cuidados de enfermagem instituídos por Florence, de higiene, de conforto e de alimentação, fazem parte da base dos cuidados de hoje, com plena evolução técnica de práticas baseadas em evidências científicas. Uma enfermagem que vê as pessoas como seres holísticos, que possuem família, cultura, têm passado e futuro e têm crenças e valores que influenciam nas experiências de saúde e doença.

Nesta "guerra" atual, a enfermagem luta contra uma doença em que inexiste possibilidade de tratamento específico, o que também nos remete ao passado, a uma singela lembrança dos tempos de Florence, em cuja época atuava nos cuidados de pacientes que também tinham doenças sem uma cura específica e, principalmente, com medicações que eram raras. Fato é que após quase dois séculos nos defrontamos, mais uma vez, com uma doença que ainda não tem tratamento específico e em que as condições de trabalho também são difíceis, porém com perpectivas diferentes.

Quase que antevendo a pandemia, 
campanhas globais pela OMS, Nursing Now e ICN foram iniciadas em 2018 para apresentar os profissionais da categoria da enfermagem como os verdadeiros protagonistas da saúde. Além de uma comemoração no dia do enfermeiro em 12 de maio de 2020 com o tema Enfermeiros: uma voz para liderar - Enfermagem do mundo e comemoração do bicentenário do nascimento de Florence Nightingale $\mathrm{e}^{(2-3,7)}$. Para além das campanhas e comemorações ao redor do mundo, um importante relatório foi emitido em abril de 2020 sobre a situação mundial da profissão de enfermagem ${ }^{(16)}$.

Cabe ressaltar que a falta de visibilidade social da enfermagem se dá, entre outros aspectos, também pela falta de reconhecimento, autonhecimento e valorização da própria categoria(6). No entanto, parece que os problemas do passado adquiriram transparência diante das medidas tomadas pelos órgãos mundiais de saúde e pelos órgãos da própria categoria marcando o presente na história da enfermagem, podendo-se inclusive afirmar que a profissão, até então quase "invisível", ganha relevância no mundo nesta pandemia. Por conseguinte, desperta-se não só a categoria para seu importante papel na sociedade, como também se promove o reconhecimento de toda população pelo seu protagonismo.

Como perspectivas para o futuro da enfermagem, é importante que se concretizem as orientações de ações destacadas no relatório, que norteiam para a necessidade de políticas para suprir as demandas oriundas de problemas do passado, abundantemente presentes nesta pandemia no mundo.

\section{CONCLUSÃO}

Nesta abordagem, conclui-se que, no mundo, a pandemia pelo SARS-CoV-2, causador da doença COVID-19, tem assolado nossas populações pela falta de um tratamento específico e estratégias globais foram necessárias para minimizar sua rápida disseminação.

Os profissionais de enfermagem, neste contexto, têm sido a linha de frente nesta pandemia tornando-se protagonistas nos cuidados de controle da infecção causada pela doença e ganhando visibilidade no mundo.

Finalmente, as perspectivas para o futuro da enfermagem consubstanciam-se na concretização das ações políticas de cada país que devem visar suprimento das demandas identificadas pelos órgãos de saúde e da categoria, evitando-se com isso a repetição de problemas do passado.

\section{Referências}

1. Organização Mundial da Saúde (OMS). Folha informativa: COVID-19 (doença causada pelo novo coronavírus) [Internet]. Brasília: OMS; 2020 [atualizado 2020 Jun 1; citado 2020 Mai 10]. Disponivel em: https://www. paho.org/bra/index.php?option=com_content\&view=article\&id=6101:covid19\&ltemid $=875$.

2. Nursing Now [Internet]. Nursing Now é um programa do Burdett Trust for Nursing 2018 [Internet]. Genebra: ICN; 2020 [citado 2020 Mai 10]. Disponível em: https://www.icn.ch/what-we-do/campaigns/nursing-now. 3. Conselho Internacional de Enfermeiras (ICN). Covid-19 e Ano Internacional da Enfermeira e da Parteira 2020 [Internet]. Genebra: ICN; 2020 [citado 2020 Mai 10]. Disponível em: https://www.2020yearofthenurse.org/.

4. Christopher J. Gill, Gillian C. Gill, Nightingale in Scutari: Her Legacy Reexamined. Clinical Infectious Diseases [Internet]. 2005 Jun [citado em 10 mai. 2020]; 40(12):1799-1805. Disponível em: https://pubmed.ncbi.nlm.nih. gov/15909269/.

5. Costa $R$, et al. 0 legado de Florence Nightingale: uma viagem no tempo. Texto contexto - enferm. [Internet]. 2009 [citado em 10 mai 2020]; 18(4): pag. 661-669. Disponivel em: https://www.scielo.br/scielo.php?pi$\mathrm{d}=$ S0104-07072009000400007\&script=sci_abstract\&tlng=pt.

6. Biblioteca Central COFEN. Enfermagem - protagonismo invisível socialmente. [Internet]. Brasília: COFEN; 2020 Jul 2019 [citado 2020 Mai 10]. Disponível em: http://biblioteca.cofen.gov.br/enfermagem-protagonismo-invisivel-socialmente/.

7. Conselho federal de Enfermagem (COFEN). Define lançamento da Campanha Nursing Now. [Internet]. Brasília: COFEN; 2020 Abr 3 [citado 2020 Mai 10]. Disponivel em: http://www.cofen.gov.br/cofen-define-lancamento-da-campanha-nursing-now_69876.html.

8. Conselho Regional de São Paulo (COREN). Covid-19: Coren-SP divulga resultados da sondagem sobre EPIs durante a pandemia. [Internet]. São Paulo; COREN; $2020 \mathrm{Abr}$ [citado 2020 Mai 10]. Disponível em: https://portal.coren-sp.gov.br/noticias/covid-19-coren-sp-divulga-resultados-da-sondagem-sobre-epis-durante-a-pandemia/.

9. Ministério da Saúde, Secretária de Vigilância em Saúde (BR). Guia de Vigilância Epidemiológica para Infecção humana para o COVID-19. [Internet].
Brasília: Ministério da Saúde; 2020. [citado 2020 Mai 10]. Disponível em: https://portalarquivos.saude.gov.br/images/pdf/2020/April/07/GuiaDeVigiEpidemC19-v2.pdf.

10. Conselho Federal de Enfermagem (COFEN). Observatório de Enfermagem. [Internet]. Brasília: COFEN; 2020 [atualizado 2020 Jun 2 ; citado 2020 Jun 2]. Disponível em: http://observatoriodaenfermagem.cofen.gov.br/. 11. Campos CJG. Content analysis: a qualitative data analysis tool in health care. Rev Bras Enferm. [Internet]. 2004 Set/Out [cited Mai,10 2020];57(5):611-4. Available from: www.scielo.br/pdf/reben/v57n5/ a19v57n5.pdf.

12. Cui J, Li F, Shi ZL. Origin and evolution of pathogenic coronaviruses. Nat Rev Microbiol. [Internet]. 2019 [cited Mai,10 2020]; 17 (3):181-192. Available from: https://www.ncbi.nlm.nih.gov/pmc/articles/PMC7097006/.

13. Agência Nacional de Vigilância Sanitária (BR). Nota técnica GVIMS/ GGTS/ANVISA n. ${ }^{\circ} 04 / 2020$. Orientações para serviços de saúde: Medidas de prevenção e controle que devem ser adotadas durante a assistência aos casos suspeitos ou confirmados de infecção pelo novo coronavírus (SARS-CoV-2).[Internet]. Brasília: ANVISA; 2020. [citado 2020 Mai 10]. Disponível em: https://www20.anvisa.gov.br/segurancadopaciente/index.php/alertas/ category/covid-19.

14. Ministério da Saúde, Secretária de Vigilância em Saúde (BR). Boletim Epidemiológico 07. Doença pelo Coronavírus (COVID-19). [Internet]. Brasília: Ministério da Saúde; 2020. [citado 2020 Mai 10]. Disponível em: https:// www.saude.gov.br/images/pdf/2020/April/06/2020-04-06-BE7-Boletim-Especial-do-COE-Atualizacao-da-Avaliacao-de-Risco.pdf.

15. World Health Organization (WHO). Situation Report 133. Coronavirus disease (COVID-19). [Internet]. Genebra:WHO; 2020 [atualizado 2020 Jun 1; citado 2020 Jun 2]. Disponível em: https://www.who.int/docs/default-source/coronaviruse/situation-reports/20200601-covid-19-sitrep-133.pdf?sfvrs$\mathrm{n}=9 \mathrm{a} 56 \mathrm{f} 2 \mathrm{ac} \_4 \& \mathrm{ua}=1$.

16. World Health Organization (WHO). State of the world's nursing. Investing in education, jobs and leadership. 2020. [Internet]. Genebra: WHO; 2020. [citado 2020 Mai 10]. Disponível em: https://www.who.int/publications-detail/nursing-report-2020. 